\title{
Acute Coronary Syndrome and Myocardial Ischemia in Hypertrophic Cardiomyopathy
}

\author{
Ambreen Gul, Ali Gohar Lodro \\ Department of Cardiology, Faculty of Medicine, Southend University Hospital, Southend, UK \\ Email address: \\ Ambreen.gul@southend.nhs.uk (A. Gul), gulambreen2001@yahoo.com (A. G. Lodro) \\ To cite this article: \\ Ambreen Gul, Ali Gohar Lodro. Acute Coronary Syndrome and Myocardial Ischemia in Hypertrophic Cardiomyopathy. Cardiology and \\ Cardiovascular Research. Vol. 4, No. 1, 2020, pp. 22-26. doi: 10.11648/j.ccr.20200401.15
}

Received: November 7, 2019; Accepted: January 29, 2020; Published: March 18, 2020

\begin{abstract}
: 47 years old male smoker with no co morbidities, presented with history of chest pain in the retrosternal area for the 3 days. The Pain was described as sharp, non-exertional and intermittent. Patient did not have any family history of known cardiac disease or sudden cardiac death. A Clinical examination failed to reveal any abnormalities. An ECG revealed deep T wave inversion in the anterolateral chest leads with left ventricular hypertrophy (LVH). Bloods analysis showed normal Complete blood count, liver function and mildly increased cardiac troponins. The Patient was admitted to the CoronaryCare Unit with a diagnosis of acute coronary syndrome. Patient was initiated dual antiplatelets, fondaparinux and high intensity atorvastatin. Following this, an echocardiogram revealed severe Left ventricular hypertrophy and reduced LV (left ventricular) cavity dimensions. Good LV systolic function with grade 3 diastolic dysfunction was noted. Coronary angiogram showed a normal right and left coronary system. Patient was diagnosed with hypertrophic cardiomyopathy. Cardiac MRI Showed severe Left ventricular hypertrophy with interventricular septum thickness $3.7 \mathrm{~cm}$ with rest of the walls hypertrophied. There was evidence of severe fibrosis of the septum, anterior and lateral wall. On the basis of severe left ventricular hypertrophy (especially septal thickness $>3.5 \mathrm{~cm}$ ) and myocardial fibrosis, Patient was started on beta blockers and ICD was inserted for primary prevention of arrhythmias.
\end{abstract}

Keywords: Hypertrophic Cardiomyopathy, Acute Coronary Syndrome, Myocardial Fibrosis

\section{Introduction}

Hypertrophic cardiomyopathy (HCM) is a genetically determined heart muscle disease most commonly caused by mutations in sarcomere genes. Its Prevalence in general population is $0.2 \%$ [1-4].

Subsequent newer techniques has increased recognition of hypertrophic cardiomyopathy (HCM) phenotype and improved clinical diagnosis. In addition, prior epidemiological studies didn't take into account autosomal dominant transmission of HCM. For these reason, Prevalence of HCM in general population has been estimated to closer to 0.5 percent [5].

Hypertrophic cardiomyopathy is characterized by left ventricular hypertrophy of various morphologies including Left ventricular outflow obstruction, apical or septal hypertrophy. Structural and functional abnormalities can yield symptoms like fatigue, dyspnoea, palpitations syncope and chest pain.
Advanced heart failure symptoms of orthopnea, paroxysmal nocturnal dyspnea, and oedema are uncommon

This case is important as the patient's presentation was highly suggestive of acute coronary syndrome because of his history, ECG changes and mild increase in troponins. As a physician, it is important to possess a wide range of experience when dealing with cases.

This is a classic example of a common presentation of a relatively uncommon disease.

Other common presentation of HCM is syncope, sudden death and arrhythmias.

This case pertains to a 47 years old male with a history of chest pain lasting for three days. Patient described the pain as sharp, intermittent, non-exertional and focused in retrosternal area. Patient clinical examination was normal. An ECG revealed deep $\mathrm{T}$ wave inversion and voltage criteria for Left ventricular hypertrophy. Blood examination revealed a mild rise in troponins.

On the basis of these symptoms, the patient was diagnosed 
with acute coronary syndrome and treatment for Non-ST elevation myocardial infarction was initiated.

The Patient underwent an echocardiogram which showed severe LVH with a small LV cavity. A diagnosis of hypertrophic cardiomyopathy was then established. Patient was risk stratified and started on beta blockers. Later, an ICD was implanted for primary prevention.

\section{Case Presentation}

47 years old male smoker presented with complain of atypical chest pain for the last 3 days. The Pain described as sharp, intermittent and not associated with exertion. The Pain did not stop him doing activities of normal living. He also mentioned mild shortness of breath upon exertion for last 2 months which he links to his heavy smoking.

No history of syncope, palpitations.

No history of any previous cardiac illness.

No history of any use of recreational drugs. The Patient was not on any medication prior to presentation.

The Patient is Taxi Driver by profession

No family history of any cardiac illness or sudden death

Clinical examination:

HR 72/min.
BP: $127 / 78 \mathrm{~mm}$ of $\mathrm{Hg}$.

Good oxygen saturation on room air.

Cardiac and chest examination were normal.

\subsection{Investigations}

1) ECG revealed deep symmetrical $T$ wave inversion (Figure 1).

2) Cardiac Troponins I: $41 \mathrm{ng} / \mathrm{L}$ with follow up level 35 $\mathrm{ng} / \mathrm{L}$.

3) Liver and renal function were normal.

4) Echocardiogram: Revealed severe concentric LVH with small size LV cavity. Interventricular septum measured $3.7 \mathrm{~cm}$ with Normal valves. Normal RV function. (Figures 2 and 3).

5) Coronary angiogram which showed normal right and left coronary system.

6) Cardiac MRI: Normal size Left Ventricular cavity with mildly impaired LV systolic function. Massive asymmetrical and nodular hypertrophy measuring $42 \mathrm{~mm}$ in mid cavity. Normal valves.

Extensive fibrosis throughout in septum, anterior and inferior wall. Myocardial mass $277 \mathrm{gm} / \mathrm{m}^{2}$ (Normal 75 $114 \mathrm{gm} / \mathrm{m}^{2}$ ) (Figures 4 and 5).

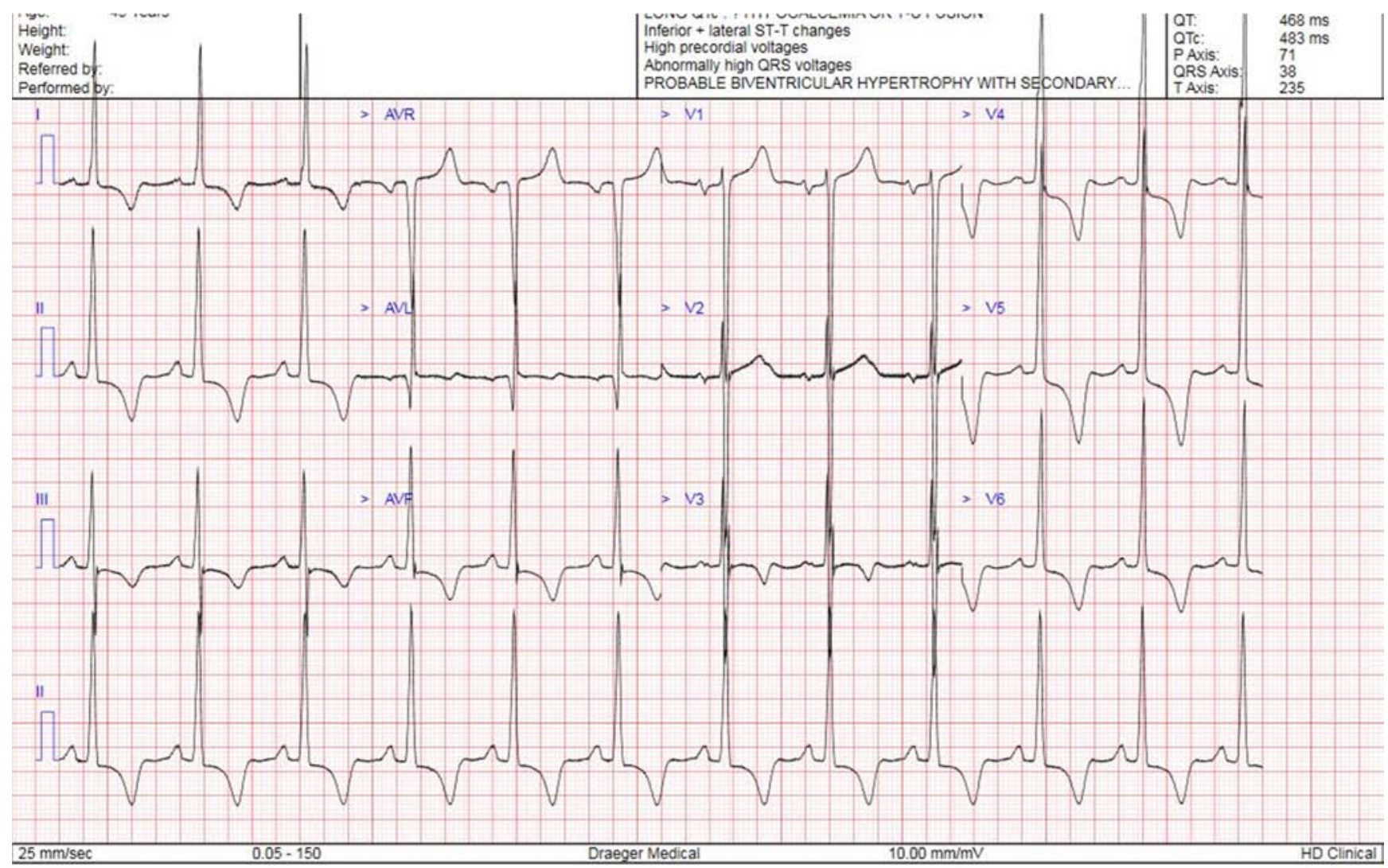

Figure 1. ECG showing deep T wave inversion in leas I, II, III, AVF and V3-V6. Voltage criteria for severe Left ventricular hypertrophy (LVH).

\subsection{Differential Diagnosis}

Based on the patient's history, the clinical examination ECG results, there are two most likely differential diagnoses,

1) Acute coronary syndrome.
2) Hypertrophic cardiomyopathy.

\subsection{Treatment}

Patient was finally diagnosed with hypertrophic 
cardiomyopathy after echocardiogram and Cardiac MRI.

Patient was risk stratified and started on bisoprolol $5 \mathrm{mg}$ po daily.

Patient has ICD implantation for primary prevention as he is high risk for developing arrhythmic cardiac death.

\subsection{Outcome and Follow-up}

Patient has OPD clinic follow up with no symptoms

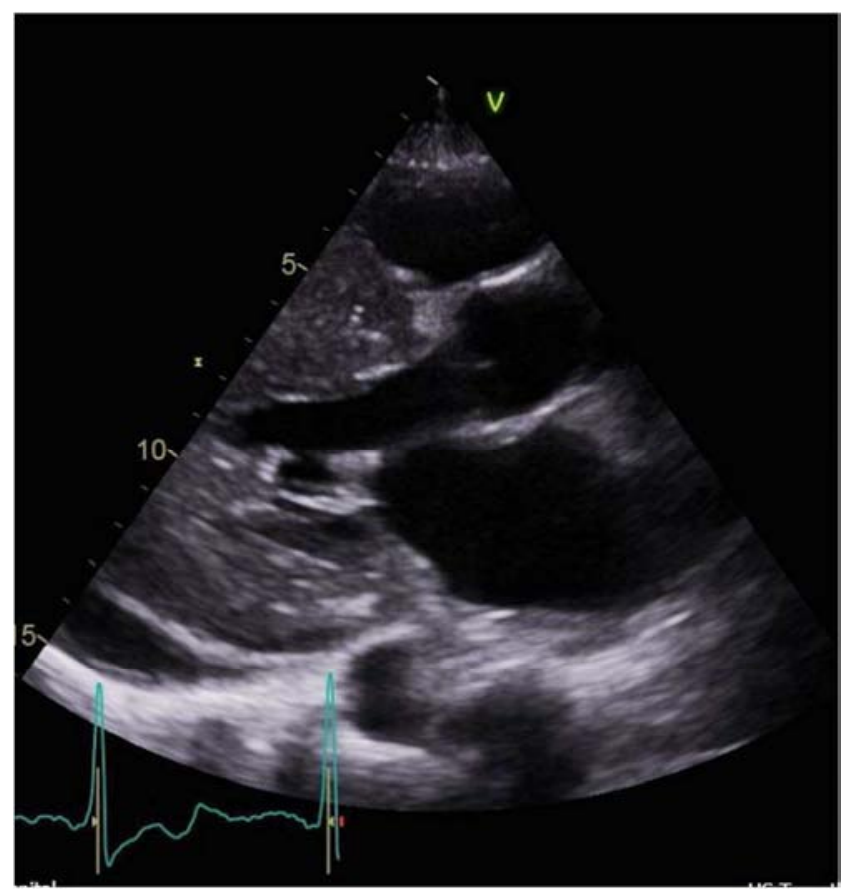

Figure 2. Echocardiogram Parasternal long axis view showing severe left ventricular hypertrophy with reduced left ventricular cavity.

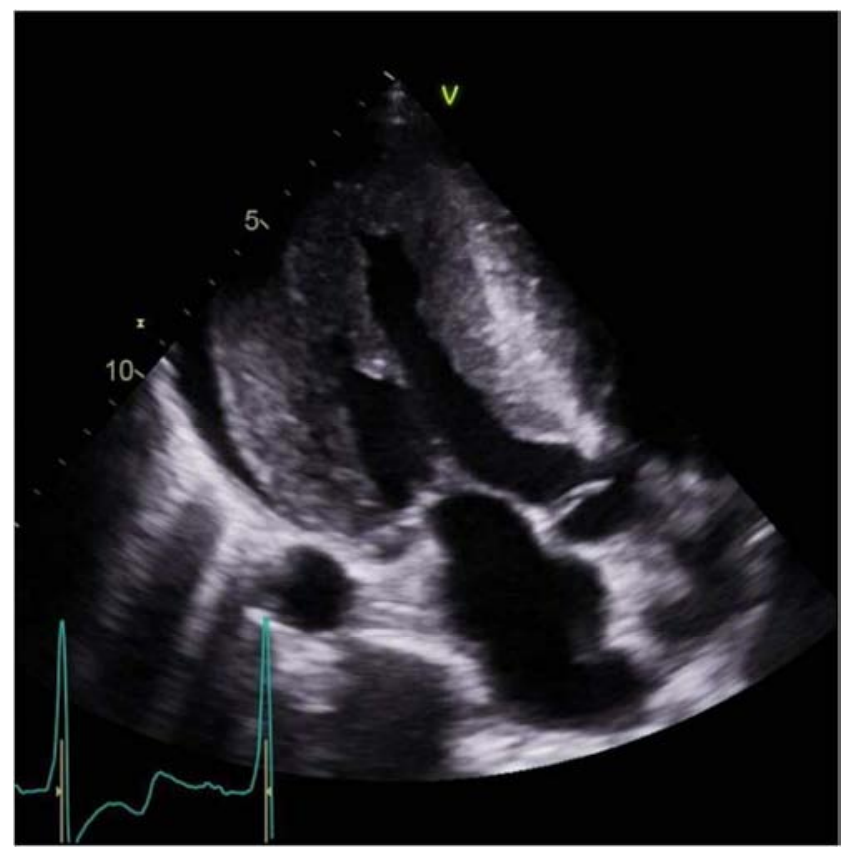

Figure 3. Echocardiogram apical long axis exhibiting hypertrophied myocardium in different view.

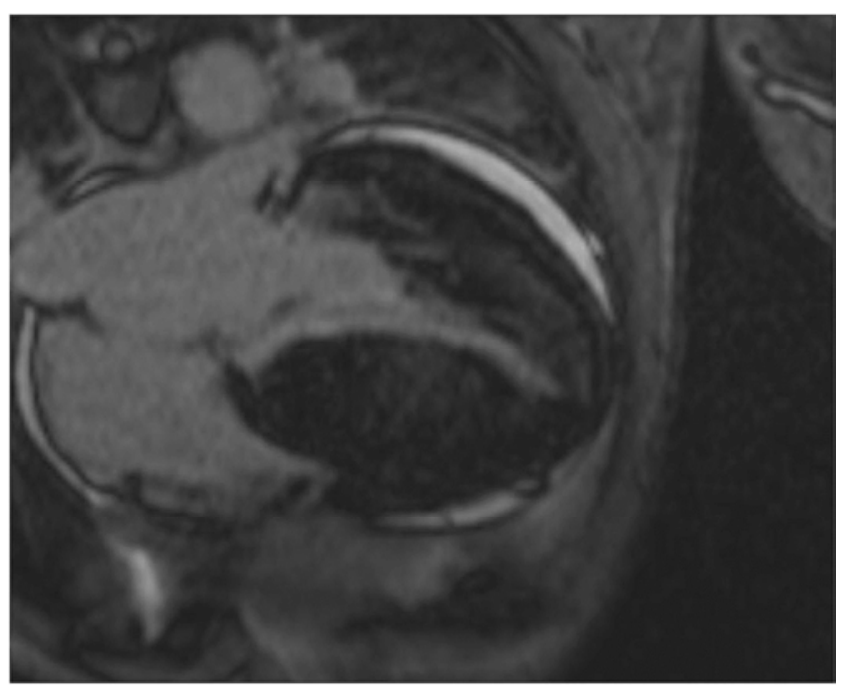

Figure 4. Myocardial resonance imaging showing more elaborate evidence of LVH.

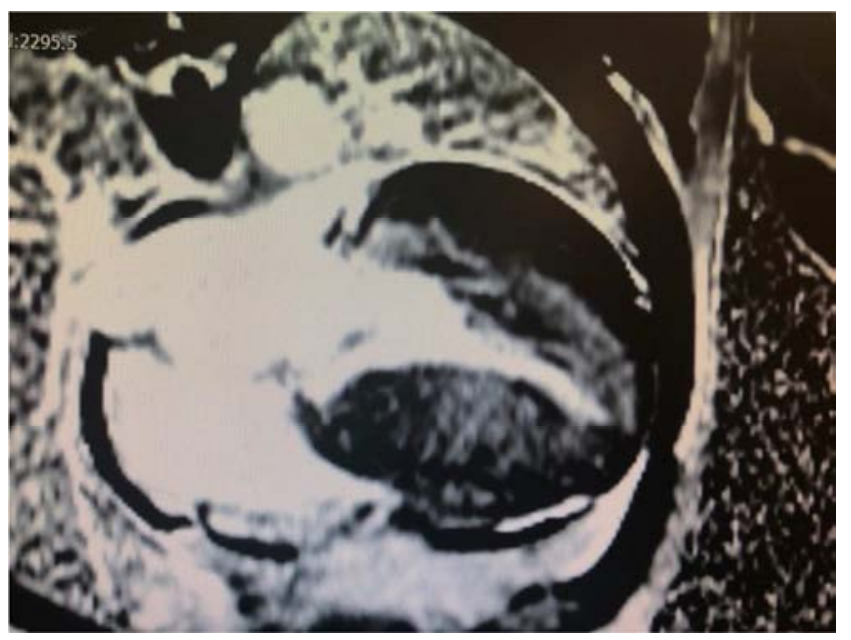

Figure 5. Late gadolinium enhancement (LGE) cardiac MRI. white areas in the myocardium shows fibrosis. Grey and dark area in left ventricle shows normal myocardiu.

\section{Discussion}

Hypertrophy Cardiomyopathy is genetic cardiomyopathy caused by mutations in sarcomere genes. Prevalence of this disease is 0.2 percent in variety of ethnic population [1-5].

Common presenting symptoms are dyspnoea on exertion, Fatigue, presyncope, syncope palpitations and chest pain.

Typical Chest pain occurs in 25 to $30 \%$ patients [6].

ECG is the most sensitive routinely performed diagnostic test for HCM but not specific. ECG changes were reported in only $5 \%$ of patients with HCM in one cohort [6-8].

Echocardiography is an important diagnostic tool. Any unexplained increase in LV wall thickness greater than $1.5 \mathrm{~mm}$ is considered to be diagnostic of HCM.

The initial diagnosis of NSTEMI in our patient appears wrong, but ischemia may coexist in patient with hypertrophic cardiomyopathy. The Pathophysiology of ischemia in HCM is different from acute coronary syndrome. 
Several potential mechanisms have been proposed to explain ischemia in patients with hypertrophic cardiomyopathy. Raphael et all, described three main causes. Firstly, abnormalities in intramyocardial arterioles and coronary flow abnormalities with flow reversal during systole and higher flow velocities during diastole cause relative ischemia.

Secondly, increase wall thickness result in supply/demand mismatch. Lastly, increased compression of microcirculation during systole contributes to ischemia [9].

Patient with HCM who have an acute myocardial infarction are more likely to present with non ST segment myocardial infarction rather than ST elevation myocardial infarction. These patient have less risk of mortality compared to patients with hypertrophic cardiomyopathy [10].

Recent research has demonstrated the incremental utility of cardiac magnetic resonance in the diagnosis, therapeutic planning, and prognostication of this disease.

Cardiac magnetic resonance provide additional information beyond that which is available from echocardiography $[11,12]$. Cardiac magnetic resonance can identify the areas of segmental LVH not reliably visualised by echocardiography and better structural abnormalities of mitral valve and papillary muscle.

Contrast-enhanced CMR with late-gadolinium enhancement (LGE) has the capability to identify areas of myocardial fibrosis/scarring with novel data demonstrating that the extent of LGE by CMR may play an important role in risk stratification of patients with HCM. Early studies demonstrated that patients with HCM and evidence of LGE on CMR have increased rates of non-sustained ventricular tachycardia on ambulatory holter monitoring compared with patients without LGE, raising the concept that LGE represents a substrate for generation of malignant ventricular arrhythmias [13].

A large multicentre study consisting of nearly 1300 patients with HCM demonstrated that LGE extent can identifying patients with increased risk of sudden death and deserve consideration of ICD placement. Extensive LGE, occupying more than $15 \%$ of LV mass, is equivalent to a twofold sudden death risk as compared with no LGE $[14,15]$

In short, hypertrophic cardiomyopathy heterogeneous group of genetically determined disease with variety of clinical presentation. Treatment concepts for HCM have now been revolutionized after the emergence of new imaging modalities. These strategies have found to reduce the incidence of sudden cardiac death.

\section{Conclusion}

1) Hypertrophic cardiomyopathy present with atypical chest pain. When interpreting the chest pain, it is important to consider other differential diagnoses such as structural heart disease as well.

2) Chest pain in hypertrophic cardiomyopathy could represent myocardial ischemia although the mechanism of ischemia differs from the plaque rupture or plaque instability observed in typical acute coronary syndrome. As a result, treatment with dual antiplatelet, anticoagulation and high intensity statins are of no value.

3) Myocardial fibrosis is an important histological finding in HCM; a Cardiac MRI is essential to establish this condition and may guide to device implantation (ICD) for primary prevention of arrhythmias.

\section{References}

[1] Maron BJ, Spirito P, Roman MJ, et al. Prevalence of hypertrophic cardiomyopathy in a population based sample of American Indian aged 51 to 77 years. Am J Cardiolo 2004; 93: 81510 .

[2] Zou Y, Song L, Wang Z, et all. Prevalence of idiopathic hypertrophic cardiomyopathy in China: a population-based echocardiographic analysis of 8080 adults. J Am Coll Cardiol 1999; 33: 1590 .

[3] Maron BJ, Gardin JM, Flack JM, et al. Prevalence of hypertrophic cardiomyopathy in a general population of young adults. Echocardiographic analysis of 4111 subjects in the CARDIA Study. Coronary Artery Risk Development in (Young) Adults. Circulation 1995; 92: 785.

[4] Veselka J, Anavekar NS, Charron P, Hypertrophic obstructive cardiomyopathy. Lancet 2017; 389: 1253.

[5] Semsarian J, Ingles J, Maron MS, et al. New perspective on the prevalence of hypertrophic cardiomyopathy. J of American College of Cardiology 2015; 65: 1249.

[6] Elliot PM, Kaski JC, Prasad K, et all. Chest pain during daily life in patients with hypertrophic cardiomyopathy: an ambulatory electrocardiographic study. Eur Heart J 1996; 17: 1056.

[7] McLeod CJ, Ackerman MJ, Nishimura RA, et all. Outcome of patients with hypertrophic cardiomyopathy and a normal electrocardiogram. J AM Coll Cardiolo. 2009; 54: 229.

[8] Rowin EJ, Maron BJ, Applebaum E, et al. Significance of false negative electrocardiograms in preparticipation screening of athletes for hypertrophic cardiomyopathy. Am J Cardiology 2012; 110: 1027.

[9] Claire E Raphael, Robert Cooper, Kim H Parker et al. Mechanism of Myocardial Ischemia in Hypertrophic Cardiomyopathy. J AM Coll cardiol VOL. 68, NO. 15, 2016.

[10] GuptaT, Harkrishnan P, Kolte d, et al. Outcomes of acute myocardial infarction in patients with hypertrophic cardiomyopathy: Am J Medicine 2015; 128: 879.

[11] Bogaet J, Olivotto I, MR imaging in Hypertrophic Cardiomyopathy: From Magnet to Bedsise. Radiology 2014; 273: 329 .

[12] Maron MS, Maron BJ. Clinical Impact of Contemporary Cardiovacular Magnetic Resonance Imaging in Hypertrophic Cardiomyopathy: Circulation 2015; 132: 292.

[13] Adabag AS, Maron BJ, Appelbaum E, et al. Occurrence and frequency of arrhythmias in hypertrophic cardiomyopathy in relation to delayed enhancement on cardiovascular magnetic resonance. J Am Coll Cardiol. 2008; 51: 1369-1374. 
[14] Chan RH, Maron BJ, Olivotto I, et al. Prognostic value of quantitative contrast-enhanced cardiovascular magnetic resonance for the evaluation of sudden death risk in patients with hypertrophic cardiomyopathy. Circulation. 2014; 130: 484-495.
[15] Maron MS, Appelbaun E, harrigan CJ, et al. Clinical profile and significance of delayed enhancement in hypertrophic cardiomyopathy. Circ Heart Fail 2008; 1; 184. 